\title{
Circulating Cytokine Profiles and Antineutrophil Cytoplasmic Antibody Specificity in Patients With Antineutrophil Cytoplasmic Antibody-Associated Vasculitis
}

\author{
Alvise Berti (iD, ${ }^{1}$ Roscoe Warner, ${ }^{2}$ Kent Johnson, ${ }^{2}$ Divi Cornec, ${ }^{3}$ Darrell Schroeder, ${ }^{4}$ Brian Kabat, ${ }^{4}$ \\ Carol A. Langford, ${ }^{5}$ Gary S. Hoffman, ${ }^{5}$ Fernanado C. Fervenza, ${ }^{4}$ Cees G. M. Kallenberg, ${ }^{6}$ Philip Seo, ${ }^{7}$ \\ Robert Spiera, ${ }^{8}$ E. William St.Clair ${ }^{9}$ Paul Brunetta, ${ }^{10}$ John H. Stone,${ }^{11}$ Peter A. Merkel, ${ }^{12}$ Ulrich Specks, ${ }^{4}$ and \\ Paul A. Monach, ${ }^{13}$ for the RAVE-ITN Research Group
}

Objective. To evaluate circulating cytokine profiles in patients with antineutrophil cytoplasmic antibody-associated vasculitis (AAV), classified by antineutrophil cytoplasmic antibody (ANCA) specificity (proteinase 3 ANCA [PR3-ANCA] versus myeloperoxidase ANCA [MPOANCA]) or by clinical diagnosis (granulomatosis with polyangiitis [GPA] versus microscopic polyangiitis [MPA]).

Methods. A panel of 29 cytokines was tested in 186 patients with active AAV at inclusion into the Rituximab in AAV trial. Cytokine concentrations were compared between groups within each classification system. Multivariable analyses adjusted for age, sex, and renal insufficiency were performed, with each biomarker as a dependent variable and ANCA specificity and clinical diagnosis as explanatory variables of interest.

Results. Levels of 9 circulating cytokines (interleukin-6 [IL-6], granulocyte-macrophage colony-stimulat- ing factor [GM-CSF], IL-15, IL-18, CXCL8/IL-8, CCL-17/thymus and activation-regulated chemokine [TARC], IL-18 binding protein [IL-18 BP], soluble IL-2 receptor $\alpha$ [sIL-2R $\alpha$ ], and nerve growth factor $\beta$ [NGF $\beta]$ ) were significantly higher in PR3-AAV than MPO-AAV, 4 cytokines (sIL6R, soluble tumor necrosis factor receptor type II [sTNFRII], neutrophil gelatinase-associated lipocalin [NGAL], and soluble intercellular adhesion molecule 1 [sICAM-1]) were higher in MPO-AAV than in PR3-AAV, 6 cytokines (IL-6, GM-CSF, IL-15, IL-18, sIL-2R $\alpha$, and NGF $\beta$ ) were higher in GPA than in MPA, and 3 cytokines (osteopontin, sTNFRII, and NGAL) were higher in MPA than in GPA (all $P<0.05$ ). For nearly all cytokines, the difference between PR3-AAV and MPO-AAV was larger than that between GPA and MPA. The multivariate analysis showed that 8 cytokines (IL-15, IL-8, IL-18 BP, NGF- $\beta$, sICAM-1, TARC, osteopontin, and kidney injury molecule 1
Supported by the Vasculitis Clinical Research Consortium, which has received support from the NIH (National Institute of Arthritis and Musculoskeletal and Skin Diseases grants U54-AR057319, RC1-AR-058303, and P60-AR-047785), the National Center for Research Resources (grant U54-RR-019497), the National Institute of Neurological Disorders and Stroke (grant NS-064808), and the Office of Rare Diseases Research. The RAVE Trial was performed with the support of the Immune Tolerance Network (NIH grant N01-AI-15416), an international clinical research consortium supported by the National Institute of Allergy and Infectious Diseases and the Juvenile Diabetes Research Foundation. Genentech and Biogen Idec provided the study medications and partial funding for the RAVE trial. At the Mayo Clinic and Foundation, the trial was supported by a Clinical and Translational Science Award from the National Center for Research Resources (NCRR) (grant RR-02415001), at Johns Hopkins University, by the NCRR (grant RR-025005) and Career Development awards K24-AR-049185 (to Dr. Stone) and K23-AR-052820 (to Dr. Seo), and at Boston University, by a Clinical and Translational Science Award (grant RR-025771), the NIH (grant M01-RR-00533), and Career Development award K24-AR-02224 (to Dr. Merkel). Dr. Monach's work was supported by the Arthritis Foundation (Arthritis Investigator Award).

${ }^{1}$ Alvise Berti, MD: Mayo Clinic College of Medicine and Science, Rochester, Minnesota, San Raffaele University, Milan, Italy, and Santa Chiara Hospital, Trento, Italy; ${ }^{2}$ Roscoe Warner, PhD, Kent
Johnson, MD: University of Michigan Medical School, Ann Arbor; ${ }^{3}$ Divi Cornec, MD, PhD: Mayo Clinic College of Medicine and Science, Rochester, Minnesota, and Université de Bretagne Occidentale, CHU de Brest, Brest, France; ${ }^{4}$ Darrell Schroeder, MS, Brian Kabat, MS, Fernanado C. Fervenza, MD, PhD, Ulrich Specks, MD: Mayo Clinic College of Medicine and Science, Rochester, Minnesota; ${ }^{5}$ Carol A. Langford, MD, MHS, Gary S. Hoffman, MD, MS: Cleveland Clinic, Cleveland, Ohio; ${ }^{6}$ Cees G. M. Kallenberg, MD, PhD: University Medical Center Groningen, Groningen, The Netherlands; ${ }^{7}$ Philip Seo, MD, MPH: Johns Hopkins University, Baltimore, Maryland; ${ }^{8}$ Robert Spiera, MD: Hospital for Special Surgery, New York, New York; ${ }^{9}$ E. William St.Clair, MD: Duke University Medical Center, Durham, North Carolina; ${ }^{10}$ Paul Brunetta, MD: Genentech, South San Francisco, California; ${ }^{11}$ John H. Stone, MD, MPH: Massachusetts General Hospital, Boston; ${ }^{12}$ Peter A. Merkel, MD, MPH: University of Pennsylvania, Philadelphia; ${ }^{13}$ Paul A. Monach, MD, PhD: Boston University and VA Boston Healthcare System, Boston, Massachusetts.

Address correspondence to Ulrich Specks, MD, Division of Pulmonary and Critical Care Medicine, Department of Internal Medicine, Mayo Clinic, College of Medicine and Science, 200 First Street SW, Rochester, MN 55905. E-mail: specks.ulrich@mayo.edu. Submitted for publication October 6, 2017; accepted in revised form February 22, 2018. 
$(P<0.05)$ distinguished patients with AAV better (lower $P$ values and larger effect sizes) when grouped by ANCA specificity than by clinical diagnosis.

Conclusion. Distinct cytokine profiles were identified for PR3-AAV versus MPO-AAV and for GPA versus MPA. Differences in these circulating immune mediators are more strongly associated with ANCA specificity than with clinical diagnosis, suggesting that heterogeneity in the AAV subtypes extends beyond clinical phenotypes.

Antineutrophil cytoplasmic antibodies (ANCAs) are the primary serologic markers of ANCA-associated vasculitis (AAV), a group of primary systemic necrotizing small vessel vasculitides that includes granulomatosis with polyangiitis (GPA) and microscopic polyangiitis (MPA) $(1,2)$. GPA and MPA share many of the clinical features induced by capillaritis. Yet it remains a matter of debate whether they represent expression of the same disease spectrum or 2 distinct condition (1). GPA differs from MPA due to the presence of necrotizing granulomatous tissue inflammation, a different organ predilection, and association with different ANCA specificity (1).

Patients with GPA are more likely to have ANCAs directed against proteinase 3 (PR3), whereas patients with MPA more often have ANCAs against myeloperoxidase (MPO), but there is substantial overlap. Recent data suggest that ANCA specificity may have stronger associations with genetic predisposition (3), response to therapy (4), relapse risk $(5,6)$, and long-term prognosis $(7)$ than clinical diagnosis does, emphasizing the clinical utility of ANCA specificity in the classification of patients with AAV.

As in other autoimmune diseases, the role of cytokines in the pathogenesis of $\mathrm{AAV}$ is now emerging (8-11). The levels of some of these circulating immune mediators have been shown to be elevated in patients with severe active AAV and to decline after treatment, distinguishing active AAV from remission better than conventional markers such as the C-reactive protein level and erythrocyte sedimentation rate (9). Even though evidence supporting classification based on ANCA specificity is now accumulating, a specific characterization of circulating cytokine profiles associated with ANCA specificity or with clinical diagnosis has not yet been performed.

Therefore, we evaluated a panel of 29 circulating immune mediators associated with inflammation, proliferation, vascular injury, and tissue damage and repair in serum samples from patients with active AAV collected at the time of inclusion into a large, prospective clinical trial and determined their association with ANCA specificity (PR3-AAV versus MPO-AAV) and clinical diagnosis (GPA versus MPA).

\section{PATIENTS AND METHODS}

Patient classification and study design. The patients included in this study were enrolled in the Rituximab in AAV (RAVE) trial, a double-blind, double-dummy-controlled trial of 197 patients with active severe GPA or MPA randomized to receive either cyclophosphamide followed by azathioprine, or rituximab (12). A positive serum assay for PR3-ANCA or MPOANCA and a Birmingham Vasculitis Activity Score for Wegener's Granulomatosis (BVAS/WG) (13) of $\geq 3$ were required for enrollment. Details of the trial design and the main study results have been published previously $(12,14)$. Of the 197 patients included in the RAVE trial, 187 had baseline serum samples available for the purpose of this study. One patient was excluded from this analysis because a clinical diagnosis of GPA versus MPA was indeterminate. Thus, data for 186 patients were included. Patients were classified by their ANCA specificity (PR3-AAV or MPO-AAV) and by their clinical diagnosis (GPA or MPA), which were provided by the RAVE trial investigators at the time of enrollment (12).

The primary aim of this study was to compare the profiles of the selected serum biomarkers studied within each classification system. The secondary aim was to determine whether ANCAbased or clinical diagnosis-based classification showed more significant differences in these circulating immune mediators.

Baseline disease characteristics. Disease activity was assessed in all patients, using the BVAS/WG, and organ manifestations present at enrollment were recorded by the study investigators, all of whom were clinicians with expertise in vasculitis. We defined baseline phenotype categories (capillaritis, granulomatosis, renal involvement, and alveolar hemorrhage) based on the BVAS/WG items recorded at the time of enrollment, as previously described (6). Specific clinical features of classification grouping (ANCA specificity and clinical diagnosis) in the RAVE cohort were analyzed in a previous study (4).

Serum sample processing and cytokine assays. Serum was processed and stored at each study site and then shipped to a central repository and subsequently to the Johnson Laboratory at the University of Michigan. All samples remained frozen at $-80^{\circ} \mathrm{C}$ until the day the assays were performed. A panel of 29 cytokines was originally compiled; these cytokines were selected for their possible role as markers of disease activity and classified roughly as molecules involved in inflammation or proliferation, chemokines, soluble receptors, markers of vascular injury, or markers of tissue damage and repair, as previously reported (9). Assays for all cytokines were performed using an antibody array (a set of miniaturized sandwich immunoassays) as previously described (9), except for BAFF, which was measured by enzymelinked immunosorbent assay using monoclonal antibodies developed at Genentech.

Data processing and statistical analysis. Data were analyzed at the Mayo Clinic. Two classification systems, ANCA specificity and clinical diagnosis, defined groups for comparison. Continuous variables are summarized as the mean \pm SD or the median and interquartile range (IQR), and nominal variables are summarized as frequencies and percentages. Baseline characteristics of the patients were compared between groups for each classification system using the Wilcoxon rank sum test for continuous variables and the chi-square test for nominal variables. The Wilcoxon rank sum test was used to compare values of each serum cytokine between groups within each classification 
system. We also used parametric methods after a $\log _{10}$ transformation of values of each biomarker with resulting values standardized to have a mean of 0 and an SD of 1 . We analyzed each cytokine individually, reporting individual $P$ values and $95 \%$ confidence intervals. Then we evaluated the relative strength of association of biomarkers with groups defined using the 2 classification systems.

In order to address the question of whether ANCA specificity (PR3-ANCA versus MPO-ANCA) and clinical diagnosis (GPA versus MPA) predict the level of each cytokine, we conducted a multivariable linear regression analysis. We performed this analysis for each cytokine with the given cytokine as the dependent variable, ANCA specificity (PR3-ANCA versus MPO-ANCA), and clinical diagnosis (GPA versus MPA) as the explanatory variables of interest, and age, sex, and renal insufficiency (glomerular filtration rate $<60 \mathrm{ml} / \mathrm{minute} / 1.73 \mathrm{~m}^{2}$ ) as covariates. Initial analyses were performed to verify the absence of ANCA-by-disease interaction effects. This model approach tries to explain the strength of the association of each biomarker with PR3-AAV or MPO-AAV and GPA or MPA. Results of the multivariable analyses were summarized by presenting the respective effect estimates ( $\mathrm{Z}$ score) and corresponding $95 \%$ confidence intervals for ANCA specificity and clinical diagnosis.

$P$ values less than 0.05 were considered significant. Multiple-comparisons correction methods were not applied in parametric, nonparametric, and multivariate analyses since each cytokine was analyzed separately and without considering a set of statistical inferences simultaneously. The 2-tailed $P$ value for each analysis is presented in the Supplementary materials, available on the Arthritis \& Rheumatology web site at http:/ onlinelibrary.wiley.com/doi/10.1002/art.40471/abstract. All analyses were performed using JMP and SAS software (SAS Institute).

\section{RESULTS}

Demographic and clinical features of the patients at enrollment. The 186 patients included 92 men and 94 women with a median age of 52 years (IQR 44-66, range 15-92), all of whom had severe disease, with a median BVAS/WG of 8 (IQR 5-10, range 3-23). Of these patients, 124 had PR3-ANCA and 62 had MPO-ANCA, whereas 140 were diagnosed as having GPA and 46 were diagnosed as having MPA. The baseline characteristics of the patients with PR3-ANCA versus those with MPOANCA and of the patients with GPA versus those with MPA are summarized in Table 1 . There was substantial overlap in clinical disease manifestations between patients classified by ANCA specificity or clinical diagnosis. Yet within each classification group (ANCA specificity and clinical diagnosis), demographic characteristics, creatinine clearance, and all phenotype categories except alveolar hemorrhage were significantly different $(P<0.05$ for all comparisons) (Table 1). BVAS/WG scores were not differently distributed across the subsets.

Comparison of blood cytokine profiles between disease categories. As previously reported, each of the cytokines tested at baseline was significantly increased in the RAVE cohort compared to healthy controls, except for RANTES, angiotensin-converting enzyme, basic fibroblast growth factor, and vascular cell adhesion molecule 1 (9). Circulating cytokine profiles differed significantly between

Table 1. Baseline characteristics of the 186 patients with AAV according to serologic and clinical classifications*

\begin{tabular}{|c|c|c|c|c|c|c|}
\hline & \multicolumn{3}{|c|}{ ANCA specificity } & \multicolumn{3}{|c|}{ AAV clinical diagnosis } \\
\hline & $\begin{array}{l}\text { PR3-AAV } \\
(\mathrm{n}=124)\end{array}$ & $\begin{array}{l}\text { MPO-AAV } \\
(\mathrm{n}=62)\end{array}$ & $P$ & $\begin{array}{c}\text { GPA } \\
(\mathrm{n}=140)\end{array}$ & $\begin{array}{c}\text { MPA } \\
(\mathrm{n}=46)\end{array}$ & $P$ \\
\hline Age at diagnosis, median (IQR) years & $51(40-60)$ & $59(47-71)$ & $<0.001$ & $51(43-60)$ & $66.5(45-72.3)$ & $<0.001$ \\
\hline Men, no. $(\%)$ & $70(56.5)$ & $22(35.5)$ & 0.004 & $76(54.3)$ & $16(34.8)$ & 0.027 \\
\hline Any capillaritis manifestation, no. $(\%) \dagger$ & $98(79.0)$ & $60(96.8)$ & 0.002 & $112(80.0)$ & $45(97.8)$ & 0.016 \\
\hline Any granulomatous manifestation, no. $(\%)$ & $93(75.0)$ & $23(37.1)$ & $<0.001$ & $106(75.7)$ & $10(21.7)$ & $<0.001$ \\
\hline Alveolar hemorrhage, no. $(\%) \S$ & $32(25.8)$ & $13(21.0)$ & 0.413 & $34(24.3)$ & $11(23.9)$ & 0.849 \\
\hline Any renal involvement, no. (\%) & $71(57.3)$ & $50(80.6)$ & 0.003 & $83(59.3)$ & $38(82.6)$ & 0.007 \\
\hline $\begin{array}{l}\text { Baseline creatinine clearance, median (IQR) } \\
\mathrm{ml} / \text { minute }\end{array}$ & $92.1(64.0-121.5)$ & $50.26(30.2-73.71)$ & $<0.001$ & $91.1(58.7-121.4)$ & $46.6(29.9-71.6)$ & $<0.001$ \\
\hline $\begin{array}{l}\text { Receiving steroids and/or immunosuppressive } \\
\text { agents at screening, no. }(\%)\end{array}$ & $64(51.6)$ & $34(54.8)$ & 0.802 & $70(50.0)$ & $28(60.9)$ & 0.266 \\
\hline Baseline BVAS/WG score, median (IQR) & $8(5-10)$ & $8(6-10)$ & 0.867 & $8(5-10)$ & $7(5-9)$ & 0.141 \\
\hline
\end{tabular}

* AAV = antineutrophil cytoplasmic antibody-associated vasculitis; ANCA = antineutrophil cytoplasmic antibody; PR3 = proteinase 3; MPO = myeloperoxidase; GPA = granulomatosis with polyangiitis; MPA = microscopic polyangiitis; IQR = interquartile range.

$\dagger$ Defined as the presence of 1 or more of the following Birmingham Vasculitis Activity Score for Wegener's Granulomatosis (BVAS/WG) items: cutaneous purpura, scleritis, retinal hemorrhage or exudate, sensorineural deafness, hematuria, red blood cell casts on urinalysis or glomerulonephritis, increase in creatinine level, alveolar hemorrhage, mesenteric ischemia, sensory peripheral neuropathy, or motor mononeuritis multiplex.

$\$$ BVAS/WG items reflecting underlying necrotizing granulomatous inflammation included mouth ulcers, retroorbital mass/proptosis, bloody nasal discharge, sinus involvement, salivary gland enlargement, subglottic inflammation, conductive deafness, other major or minor ear, nose, and throat involvement, pulmonary nodule/cavity, endobronchial involvement, meningitis, and cord lesion.

\& A patient was categorized as having alveolar hemorrhage only if that item was scored on the BVAS/WG.

If Patients were considered to have renal disease if any renal item on the BVAS/WG (hematuria, red blood cell casts or glomerulonephritis, increase in creatinine level, or "other") was scored. 

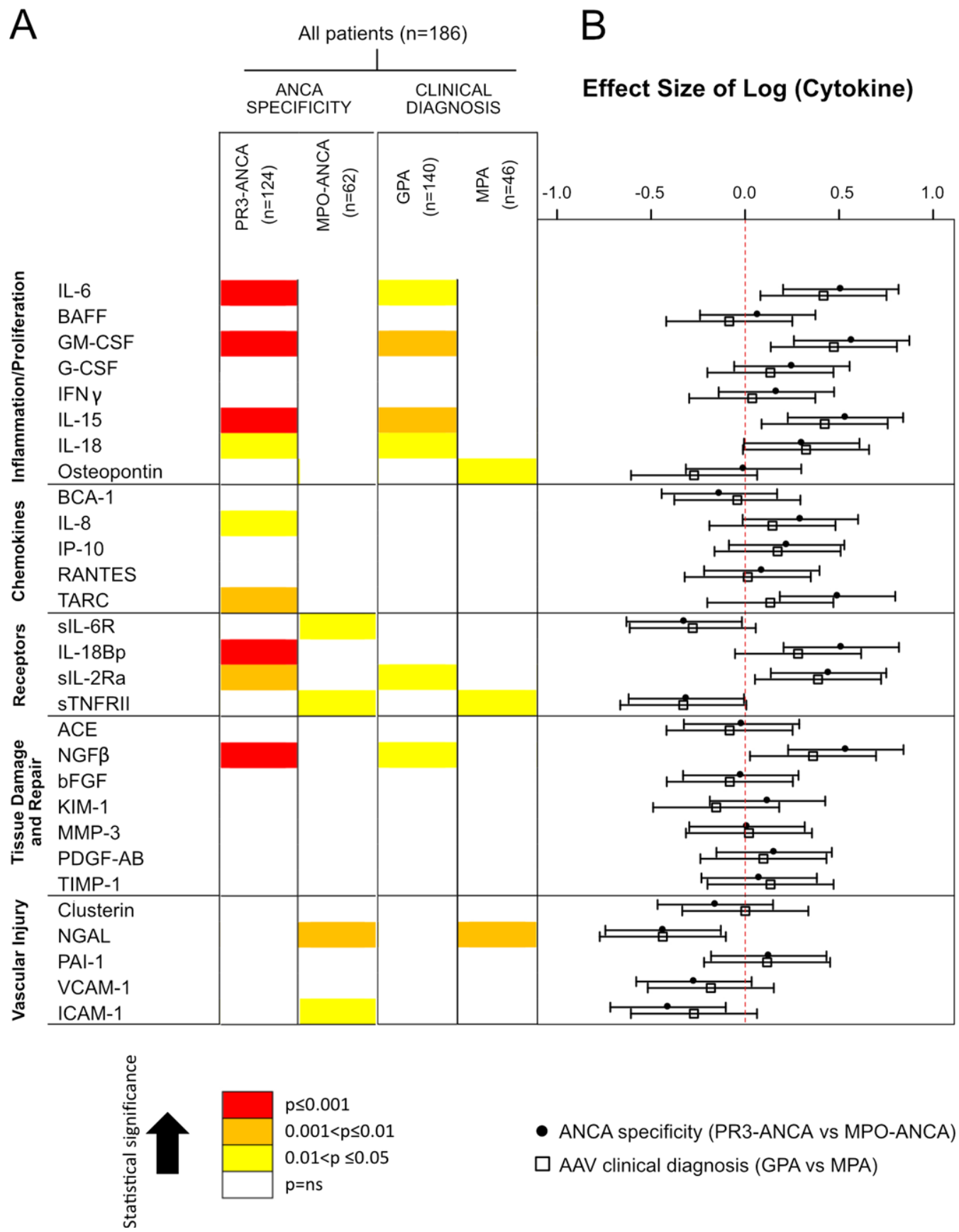

- ANCA specificity (PR3-ANCA vs MPO-ANCA)

$\square$ AAV clinical diagnosis (GPA vs MPA)

Figure 1. Association of circulating cytokines with antineutrophil cytoplasmic antibody (ANCA) type and ANCA-associated vasculitis (AAV) clinical diagnosis subgroups (ANCA against proteinase 3 [PR3-ANCA] versus ANCA against myeloperoxidase [MPO-ANCA] and granulomatosis with polyangiitis [GPA] versus microscopic polyangiitis [MPA]). A, Graphic representation of circulating cytokine profiles. Colors represent the strength of association in each classification system. B, Parametric analyses of the biomarkers (effect size) (see Patients and Methods for details). Values are the median (interquartile range). Broken line represents 0. IL-6 = interleukin-6; GM-CSF = granulocyte-macrophage colony-stimulating factor; G-CSF $=$ granulocyte colony-stimulating factor; IFN $\gamma=$ interferon- $\gamma$; BCA-1 = B cell-attracting chemokine 1; IP-10 = interferon- $\gamma$-inducible 10-kd protein; TARC $=$ thymus and activation-regulated chemokine; sIL-6R $=$ soluble IL-6 receptor; IL-18 $\mathrm{BP}=\mathrm{IL}-18$ binding protein; $\mathrm{sTNFRII}=$ soluble tumor necrosis factor receptor type II; ACE = angiotensin-converting enzyme; NGF $\beta=$ nerve growth factor $\beta$; bFGF = basic fibroblast growth factor; KIM-1 = kidney injury molecule 1; MMP-3 = matrix metalloproteinase 3; PDGF-AB = platelet-derived growth factor A and B; TIMP-1 = tissue inhibitor of metalloproteinases 1; NGAL = neutrophil gelatinase-associated lipocalin; PAI-1 = plasminogen activator inhibitor 1; VCAM-1 = vascular cell adhesion molecule 1; ICAM-1 = intercellular adhesion molecule 1; NS = not significant. 
patients with PR3-AAV and those with MPO-AAV (Figure 1A and Supplementary Table 1, available on the Arthritis \& Rheumatology web site at http://onlinelibrary.wiley. com/doi/10.1002/art.40471/abstract). Levels of 9 proteins were higher in patients with PR3-AAV than in patients with MPO-AAV (interleukin-6 [IL-6], granulocytemacrophage colony-stimulating factor [GM-CSF], IL-15, IL-18, CXCL8/IL-8, CCL17/thymus and activation-regulated chemokine [TARC], IL-18 binding protein [IL-18 $\mathrm{BP}]$, soluble IL-2 receptor $\alpha$ [sIL-2R $\alpha$ ], and nerve growth factor $\beta[\mathrm{NGF} \beta]$ ), whereas 4 biomarkers were higher in patients with MPO-AAV than in patients with PR3-AAV (sIL-6R, soluble tumor necrosis factor receptor type II [sTNFRII], neutrophil gelatinase-associated lipocalin [NGAL], and soluble intercellular adhesion molecule 1 [sICAM-1]).

In contrast, the same biomarkers were less often associated with either GPA or MPA when patients were classified according to clinical diagnosis (Figure 1A and Supplementary Table 1). Serum levels of 6 markers were higher in patients with GPA than in those with MPA (IL-6, GM-CSF, IL-15, IL-18, sIL-2R $\alpha$, and NGF $\beta$ ), and 3 were higher in patients with MPA than in patients with GPA (osteopontin, sTNFRII, and NGAL). Thus, more cytokines were associated with either PR3-AAV or MPOAAV than with either GPA or MPA. The difference between biomarker concentrations was also greater for PR3-ANCA versus MPO-ANCA than for GPA versus MPA in 9 cases (Figures 1A and B). Patients with PR3AAV had the highest number of significantly associated serum biomarkers, most of them with a stronger association when compared to other subsets (Figure 1).

We also compared cytokine levels among subgroups of patients with AAV by combining ANCA specificity and clinical phenotype, namely patients with GPA with PR3-ANCA $(\mathrm{n}=121)$, patients with MPA with PR3ANCA $(\mathrm{n}=3)$, patients with GPA with MPO-ANCA $(\mathrm{n}=$ $19)$, and patients with MPA with MPO-ANCA $(n=43)$. The serum levels of 10 of 29 cytokines differed significantly between patients with GPA with PR3-ANCA and patients with GPA with MPO-ANCA, serum levels of 2 cytokines differed significantly between patients with GPA with MPO-ANCA and patients with MPA with MPO-ANCA (i.e., kidney injury molecule 1 [KIM-1] and osteopontin), and serum levels of 12 cytokines differed significantly between patients with GPA with PR3-ANCA and patients with MPA with MPO-ANCA (see Supplementary Table 2, available on the Arthritis \& Rheumatology web site at http:// onlinelibrary.wiley.com/doi/10.1002/art.40471/abstract).

Association of cytokines with ANCA specificity and clinical diagnosis. To evaluate how strongly each cytokine is associated with either ANCA specificity or clinical diagnosis, a multivariable analysis directly comparing each classification system was performed. From this analysis 8 biomarkers were found to have significant independent multivariable associations with ANCA specificity and/or clinical diagnosis (Figure 2A and Supplementary Table 3 and Supplementary Figure 1, available on the Arthritis \& Rheumatology web site at http://onlinelibrary.wiley.com/ doi/10.1002/art.40471/abstract). For 5 biomarkers there was an independent association with ANCA specificity only. IL-18 BP, NGF, IL-8, and IL-15 were associated with PR3-AAV, and sICAM-1 was associated with MPO-AAV. For 3 biomarkers there was a significant association with both ANCA specificity and clinical diagnosis. Osteopontin, KIM-1, and TARC were all associated with PR3-AAV and MPA. No biomarker was found to have a significant association with clinical diagnosis alone. Figure 2B illustrates the relative capacity of the 2 classification systems to simultaneously explain the values of all biomarkers tested.

\section{DISCUSSION}

The results of this exploratory analysis conducted in patients with severe active AAV suggest that circulating serum cytokines reflect ANCA specificity better than they reflect clinical diagnosis. Using a panel of 29 circulating cytokines that have already been shown to be individually associated with AAV disease activity or implicated in its pathogenesis $(8,9)$, we demonstrated that these molecules are more strongly related to ANCA specificity (PR3-AAV versus MPO-AAV) than to clinical diagnosis (GPA versus MPA).

This study identified distinct cytokine profiles for PR3-AAV versus MPO-AAV and for GPA versus MPA, with a higher number of cytokines associated with and a larger effect size in favor of PR3-AAV than MPO-AAV, GPA, or MPA (Figure 1). These findings indirectly suggest that certain combinations of pathways might be more involved in PR3-AAV than in GPA, MPA, and MPOAAV. For instance, signaling cascades critical for proliferation or survival of PR3-ANCA-producing B cells may drive or potentially be impacted by this cytokine network $(11,15)$.

Since different subsets of patients showed distinctive cytokine profiles, our results suggest that different targeted treatment approaches could be evaluated separately in clinical trials for the different subsets of $\mathrm{AAV}$, similar to other autoimmune diseases in which different cytokine profiles correspond to different disease activity and treatment responsiveness (16). Our study had low power to detect differences in subgroups defined by the combination of ANCA specificity and clinical phenotype, and we were unable to compare cytokine levels in the subgroup with 
A
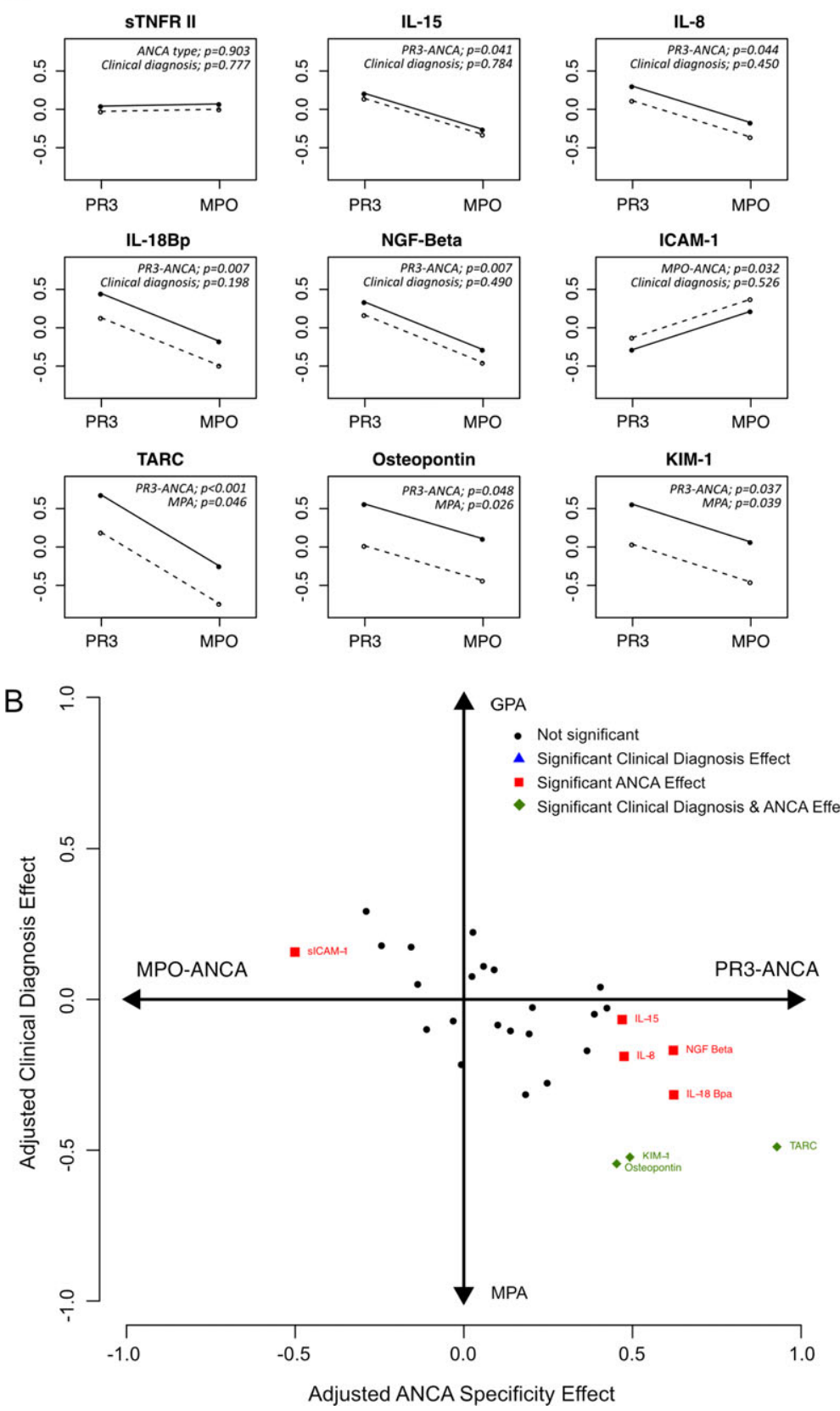

Figure 2. Multivariate analysis comparing the 2 classification systems (PR3-ANCA versus MPO-ANCA and GPA versus MPA) for each cytokine. A, Associations of each biomarker with ANCA specificity and clinical diagnosis. One example of a molecule not associated with either ANCA specificity or clinical diagnosis (sTNFRII) is shown. The other panels show the 8 soluble mediators with a significant association with ANCA type and/or clinical diagnosis. The magnitude of the difference between MPA (solid lines) and GPA (broken lines) is visually depicted by the distance between the 2 lines. The magnitude of the difference between PR3-ANCA and MPO-ANCA is visually represented by the slope of the lines. The statistical model forces them to be parallel. The direction and the grade of inclination represent the type and the strength of the association with ANCA type, respectively. B, Scatterplot comparing the effect of ANCA type and clinical diagnosis for all serum cytokines studied. The more a biomarker is skewed to the left or right, the more strongly it discriminates patients by ANCA type. The more a biomarker is skewed to the bottom or top, the more strongly it discriminates patients by clinical diagnosis. See Figure 1 for definitions. Color figure can be viewed in the online issue, which is available at http:// onlinelibrary.wiley.com/doi/10.1002/art.40471/abstract. 
PR3-ANCA and MPA because it comprised only 3 patients. Nevertheless, the differences observed between the PR3-ANCA and MPO-ANCA subgroups were also seen when patients with GPA with PR3-ANCA were compared to patients with GPA with MPO-ANCA, consistent with the main observation that cytokine profiles are more closely related to ANCA specificity than to disease phenotype. Further functional studies are needed to elucidate the interrelationships between these circulating molecules and the pathophysiologic or protective mechanisms in which they participate.

The multivariable analysis directly comparing each classification system identified 8 cytokines separating PR3-AAV from MPO-AAV. Three of them (KIM-1, TARC, and osteopontin) were also associated with clinical diagnosis and specifically with MPA. Intriguingly, no cytokines were associated with clinical diagnosis (either GPA or MPA) only, thus suggesting that these circulating immune mediators are better distinguished by ANCA specificity than by clinical diagnosis. In clinical practice, establishing an unequivocal diagnosis of GPA or MPA is often challenging for a variety of reasons, including incomplete disease manifestations at the time of the first diagnosis and disagreements between experts about the application of different definition schemes during the diagnostic evaluation of individual patients. In contrast, information on ANCA specificity is readily available and usually does not change during patient follow-up (1). Our findings provide a molecular basis supporting the concept of an ANCA-based classification of AAV, which has already been shown to convey useful information about clinical outcomes and prognoses $(1,4,5)$.

The difference, if any exists, between PR3ANCA-mediated inflammation and MPO-ANCAmediated inflammation has not yet been entirely characterized, unlike for inflammation in GPA versus MPA. Histopathologic differences in inflammation between GPA and MPA are striking, and would have suggested that cytokines correlate with phenotype rather than ANCA specificity, since serum cytokines may be considered bona fide surrogates of the immunopathologic events occurring in AAV. Therefore, our findings represent the first formal demonstration of combinations of cytokine pathways differently activated in distinct AAV subsets, and particularly in ANCA-based subsets. This study has several strengths. First, the study cohort was recruited though a stringent clinical trial protocol at centers with expertise in the study of AAV. Second, the specimens were collected, handled, and studied using strict protocols, and the assays were performed in a single laboratory by personnel who were blinded with regard to group assignment.
This study also has limitations to be considered when interpreting the findings and implications of the work. First, we acknowledge that the absence of detailed data on recent glucocorticoid treatment is a limitation. The use of glucocorticoids as first treatment for active disease before blood sampling at the time of screening may thus have influenced our results in individual patients. However, recent treatment was not significantly different in each subgroup at the screening visit (Table 1). Moreover, we had previously shown that glucocorticoid treatment prior to obtaining the blood sample had no significant detectable effect on the levels of the majority of the cytokines (9), allowing us to conclude that the differences we observed in our study among distinct AAV subsets were not driven by the effects of glucocorticoids.

Second, patients with AAV without ANCA and those with non-severe disease activity were excluded from the RAVE trial, and our findings cannot therefore be generalized to all patients with GPA or MPA. However, recent evidence supports the notion that patients with AAV who are consistently ANCA-negative may represent different subsets of disease with different pathogenesis (1).

Third, the comparison of GPA to MPA had lower statistical power to detect differences than did the comparison of PR3-AAV to MPO-AAV, due to the greater imbalance in the number of patients in the clinical diagnosis groups. However, the comparison of PR3-AAV and MPO-AAV had not only lower $P$ values, but also larger estimated effect sizes than the comparison by clinical diagnosis, highlighting the strength of our findings.

Finally, given the non-comprehensive and relatively limited number of cytokines studied, we were not able to comprehensively investigate possible mutual interactions or effects of the different cytokine pathways, thus not providing a pathophysiologic explanation of our results. The biomarkers tested were not specifically selected to represent pathophysiologic processes considered likely to differentiate subsets of AAV, and a replication of these results in an independent cohort of patients with AAV is recommended. The understanding of the reciprocal influence of these mediators is beyond the scope of our study.

In conclusion, this analysis supports the concept of an ANCA-based classification of AAV by showing that a set of selected serum biomarkers associates more strongly with either PR3-AAV or MPO-AAV than with GPA or MPA. Distinct cytokine profiles were identified for PR3AAV versus MPO-AAV and for GPA versus MPA. Differences in these circulating immune mediators are more strongly associated with ANCA specificity than with clinical diagnosis, suggesting that heterogeneity in the AAV subtypes extends beyond the clinical phenotypes identified 
by the conventional clinical classification (GPA versus MPA). Our results provide additional support for stratification of patients by ANCA specificity for treatment trials.

\section{AUTHOR CONTRIBUTIONS}

All authors were involved in drafting the article or revising it critically for important intellectual content, and all authors approved the final version to be published. Dr. Berti had full access to all of the data in the study and takes responsibility for the integrity of the data and the accuracy of the data analysis.

Study conception and design. Berti, Cornec, Merkel, Specks, Monach. Acquisition of data. Berti, Cornec, Merkel, Specks, Monach.

Analysis and interpretation of data. Berti, Warner, Johnson, Cornec, Schroeder, Kabat, Langford, Hoffman, Fervenza, Kallenberg, Seo, Spiera, St.Clair, Brunetta, Stone, Merkel, Specks, Monach.

\section{ADDITIONAL DISCLOSURES}

Author Brunetta is an employee of Genentech.

\section{REFERENCES}

1. Cornec D, Cornec-Le Gall E, Fervenza FC, Specks U. ANCAassociated vasculitis: clinical utility of using ANCA specificity to classify patients. Nat Rev Rheumatol 2016;12:570-9.

2. Jennette JC, Falk RJ, Bacon PA, Basu N, Cid MC, Ferrario F, et al. 2012 revised International Chapel Hill Consensus Conference nomenclature of vasculitides. Arthritis Rheum 2013;65:1-11.

3. Lyons PA, Rayner TF, Trivedi S, Holle JU, Watts RA, Jayne DR, et al. Genetically distinct subsets within ANCA-associated vasculitis. N Engl J Med 2012;367:214-23.

4. Unizony S, Villarreal M, Miloslavsky EM, Lu N, Merkel PA, Spiera R, et al. Clinical outcomes of treatment of anti-neutrophil cytoplasmic antibody (ANCA)-associated vasculitis based on ANCA type. Ann Rheum Dis 2016;75:1166-9.

5. Hogan SL, Falk RJ, Chin H, Cai J, Jennette CE, Jennette JC, et al. Predictors of relapse and treatment resistance in antineutrophil cytoplasmic antibody-associated small-vessel vasculitis. Ann Int Med 2005;143:621-31.
6. Fussner LA, Hummel AM, Schroeder DR, Silva F, Cartin-Ceba R, Snyder MR, et al. Factors determining the clinical utility of serial measurements of antineutrophil cytoplasmic antibodies targeting proteinase 3. Arthritis Rheumatol 2016;68:1700-10.

7. Tanna A, Guarino L, Tam FW, Rodriquez-Cubillo B, Levy JB, Cairns TD, et al. Long-term outcome of anti-neutrophil cytoplasm antibody-associated glomerulonephritis: evaluation of the international histological classification and other prognostic factors. Nephrol Dial Transplant 2015;30:1185-92.

8. Monach PA, Tomasson G, Specks U, Stone JH, Cuthbertson D, Krischer J, et al. Circulating markers of vascular injury and angiogenesis in antineutrophil cytoplasmic antibody-associated vasculitis. Arthritis Rheum 2011;63:3988-97.

9. Monach PA, Warner RL, Tomasson G, Specks U, Stone JH, Ding $\mathrm{L}$, et al. Serum proteins reflecting inflammation, injury and repair as biomarkers of disease activity in ANCA-associated vasculitis. Ann Rheum Dis 2013;72:1342-50.

10. Berti A, Cavalli G, Campochiaro C, Guglielmi B, Baldissera E, Cappio S, et al. Interleukin-6 in ANCA-associated vasculitis: rationale for successful treatment with tocilizumab. Semin Arthritis Rheum 2015;45:48-54.

11. Lepse N, Land J, Rutgers A, Kallenberg CG, Stegeman CA, Abdulahad WH, et al. Toll-like receptor 9 activation enhances B cell activating factor and interleukin-21 induced anti-proteinase 3 autoantibody production in vitro. Rheumatology (Oxford) 2016;55: $162-72$.

12. Stone JH, Merkel PA, Spiera R, Seo P, Langford CA, Hoffman GS, et al. Rituximab versus cyclophosphamide for ANCA-associated vasculitis. N Engl J Med 2010;363:221-32.

13. Stone JH, Hoffman GS, Merkel PA, Min YI, Uhlfelder ML, Hellmann DB, et al, for the International Network for the Study of the Systemic Vasculitides (INSSYS). A disease-specific activity index for Wegener's granulomatosis: modification of the Birmingham Vasculitis Activity Score. Arthritis Rheum 2001;44: 912-20.

14. Specks U, Merkel PA, Seo P, Spiera R, Langford CA, Hoffman GS, et al. Efficacy of remission-induction regimens for ANCAassociated vasculitis. N Engl J Med 2013;369:417-27.

15. Cornec D, Berti A, Hummel A, Peikert T, Pers JO, Specks U. Identification and phenotyping of circulating autoreactive proteinase 3-specific B cells in patients with PR3-ANCA associated vasculitis and healthy controls. J Autoimmun 2017;84:122-31.

16. Ronnblom L, Eloranta ML. The interferon signature in autoimmune diseases. Curr Opin Rheumatol 2013;25:248-53. 